\title{
SCALING AND MACHINE LEARNING ANALYSIS OF TURBULENT FLUXES OF MOMENTUM AND HEAT IN THE MICROCLIMATE OF AN URBAN CANYON
}

\author{
Mohsen Moradi ${ }^{1}$, William D. Lubitz ${ }^{2}$, Amir A. \\ Aliabadi* \\ School of Engineering \\ University of Guelph \\ Guelph, Canada \\ ${ }^{1}$ moradim@uoguelph.ca, ${ }^{2}$ wlubitz@uoguelph.ca \\ * Corresponding Author: aliabadi@uoguelph.ca
}

\author{
E. Scott Krayenhoff \\ School of Environmental Sciences \\ University of Guelph \\ Guelph, Canada \\ skrayenh@uoguelph.ca
}

\begin{abstract}
Turbulent flow inside the urban roughness sublayer, despite its complexities, plays a crucial role in the microclimate of the built environment. The parameterization of flow in the urban roughness sublayer provides a better understanding of turbulent exchange process leading to accurate weather forecasting. This study focused on developing relationships between turbulent quantities, including momentum and heat fluxes, and mean quantities such as mean wind speeds. Field data, including wind directions, wind speeds, and thermal stability conditions, were collected from an urban canopy in Guelph, Ontario, Canada during the summer 2017. Comparative data was obtained from a nearby rural station. A systematic scaling analysis was performed to identify a range of quantities highly related to turbulent fluxes. All combinations of quantities leading to dimensionless groups were evaluated. Linear and nonlinear correlation coefficients between different groups of variables identified when mean and turbulent quantities were related. Significant improvement in correlation coefficients was observed using high order polynomial regression, revealing the challenge of developing a robust model for predicting nonlinear behavior of turbulence. This study also used artificial neural networks (ANNs) to find nonlinear relationships between turbulent and mean quantities. As used here, an ANN is a multivariable function which attempts to approach the exact value of turbulent flux based on independent variables, properly chosen from dimensionless groups. Results showed that these approaches can successfully relate most, but not all, turbulent quantities to mean quantities.
\end{abstract}

Keywords-component; microclimate, urban canopy; turbulence; artificial neural network; scaling

\section{INTRODUCTION}

Flow interactions between near-surface regions and the atmosphere in built-up areas cause spatial and temporal flow complexity leading to phenomena such as channeling flow, secondary vortices, complex three-dimensional patterns and turbulence. Within the lower level of the urban atmosphere, which is the region from the surface up to the top of the atmospheric roughness sublayer, net transport of energy and pollutants is greatly influenced by the turbulent flow. Therefore, a general understanding of turbulent exchange processes in the lower urban atmosphere can give insight into underlying flow features. Numerous studies have investigated flow characteristics in mesoscale models that characterize large spatial and temporal scales. Three-dimensional effects, which are significant up to two or more times building heights, and the methods used for approximating the atmospheric roughness sublayer, can considerably change modeled flow and dispersion processes. Another important feature of flow is the formation of shear layers at roof level, which oscillate at dominant frequencies due to instabilities. Turbulence transport into the canopy is driven by these shear layers and turbulence characteristics vary by any change in the upwind conditions. More flow complexity arises in canopies with unequal building heights. Side separation layers, jetting flow from the windward face of downstream buildings, and channeling flow in a finite street length, can all cause additional difficulties when investigating in-canyon flows in these environments [1-4]. Analytical and numerical studies focused on the UCL are not developed enough to capture all of the important features of flow in these regions. It has been suggested that empirical relationships can be developed for the variables of interest. Turbulent exchange processes are usually investigated by measuring momentum and heat fluxes. Similarity theory is used to parameterize variables and guide the design of experiments to record the most desirable information [5]. Successful use of similarity theory requires guessing relevant variables that can be determined from measureable parameters, and ensuring these are relevant to predicting the unknown variables of interest. Numerous studies have employed Monin-Obukhov similarity theory to estimate wind, temperature, and turbulence statistics profiles as functions of friction velocity and length scales [6]. Additionally, accurate and reliable measurements of meteorological quantities, which can be obtained from field measurements or approximated from wind tunnel studies, are required to relate turbulent fluxes to other known quantities in the flow such as mean velocity or temperature. There have been many attempts to parametrize and describe turbulent transport in rough but horizontally homogenous environments. However, turbulence characteristics are still not well understood in 
horizontally inhomogeneous urban areas and further investigations are required. The pioneering efforts of Roth and Oke [7], followed by many others, have shown how to parametrize turbulent fluxes in horizontally inhomogeneous built areas. Most of these studies attempted to find or modify correlations between turbulent quantities and Monin-Obukhov length over the canopy. It has been found that there can be strong nonlinear relationships between different quantities within the canyon [8].

\section{OBJECTIVE}

This study investigated the relationships among different turbulent and mean quantities within and above an urban canyon under all wind directions, wind speeds, and thermal stability conditions. Scaling analyses were completed to identify relationships between variables. The study used measured microclimate data collected around an urban canyon in Guelph, Ontario, Canada during the summer 2018. The canyon has an aspect ratio of unity and is located on the University of Guelph campus. Strong nonlinear relationships between turbulent and mean quantities were observed. These were investigated by fitting high order polynomial equations to the data set. Artificial neural networks (ANNs) were also employed to find how turbulent fluxes can be related to groups of known variables to allow investigation of non-linear relationships. The approach presented here can be extended to turbulent flow in other canopies with different morphologies and climates.

\section{OBSERVATION SITE}

Data was collected from an urban microclimate field campaign held at the University of Guelph, Guelph, Ontario, Canada from 13th to 25th of August 2017 (Fig. 1). Meteorological conditions, including fluctuating velocity, surface and atmospheric temperature values, were measured within the urban canyon and on the roof-top of the building situated at the southwest canyon wall, using RM Young 81000 three-dimensional sonic anemometers recording at 4 and $32 \mathrm{~Hz}$. Data was also collected from the Guelph Turfgrass Institute, an open-field station representative of rural conditions located 1.9 $\mathrm{km}$ northeast of the canyon site [9]. Since local wind direction can significantly affect turbulence quantities and flow patterns within and above the urban canopy, the field data were collected and analyzed under all wind directions and thermal stability conditions.

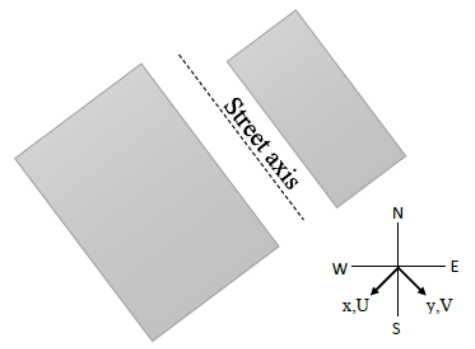

Figure 1: Top view of urban site

\section{METHODOLOGY}

Most variables will be considered to have mean and fluctuating components: $X=\bar{X}+x$ where $x$ is the turbulent fluctuating component and the overbar notation signifies a 30 minute time average of a variable. Lowercases are used to expresses variances and fluxes, e.g. $\overline{u^{2}}$ represents velocity variance in the $\mathrm{x}$ direction. Subscripts $R, S$ and $t$ denote rooflevel, street-level, and rural area measurements. ANNs, which can discover nonlinear relationships between various parameters, were employed to relate turbulent quantities

TABLE I. SCALING OF TURBULENT FLUXXS AT STREET (LEFT) AND ROOF (RIGHT) LEVELS

\begin{tabular}{|c|c|c|c|c|c|c|}
\hline \multirow{3}{*}{ 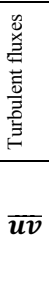 } & \multicolumn{6}{|c|}{$\begin{array}{c}\text { Urban Flow Variables } \\
\text { Correlation Coefficient (Co) }\end{array}$} \\
\hline & \multicolumn{2}{|c|}{ Street level } & \multicolumn{4}{|c|}{ Roof level } \\
\hline & $\begin{array}{c}{\overline{v^{2}}}_{S} \\
\mathrm{Co}=0.90\end{array}$ & $\begin{array}{c}{\overline{v w_{S}}} \\
\text { Co }=0.96\end{array}$ & $\begin{array}{l}{\overline{V_{S}}}^{2},{\overline{W_{R}}}^{2}, u_{* R}{ }^{2} \\
\overline{v w}_{R},{\overline{w^{2}}}_{R},{\overline{S_{R}}}^{2} \\
0.2<\text { Co }<0.4\end{array}$ & $\bar{S}$ & $\begin{array}{l}\bar{u}_{R}^{2}, \bar{v}_{R}^{2}, T K \\
0.4<\text { Co }<0.5\end{array}$ & \\
\hline$\overline{u w}$ & $\begin{array}{c}{\overline{U_{S}}}^{2},{\overline{S_{S}}}^{2} \\
0.8<\text { Co }<0.9\end{array}$ & $\begin{array}{c}{\overline{W_{S}}}^{2}, u_{* S}{ }^{2}, \bar{u}^{2} \\
\bar{w}_{S}^{2} \\
0 . T K E_{S} \\
0.9<\text { Co }<1.0\end{array}$ & $\begin{array}{c}\bar{U}_{R}^{2},{\overline{V_{R}}}^{2},{\overline{S_{t}}}^{2} \\
{\overline{U_{S}}}^{2},{\overline{S_{S}}}^{2},{\overline{v^{2}}}_{S} \\
0.2<\text { Co }<0.4\end{array}$ & $\begin{array}{c}{\overline{V_{S}}}^{2},{\overline{v^{2}}}_{R}, T K E_{R} \\
0.4<\mathrm{Co}<0.5\end{array}$ & $\begin{array}{l}u_{* R}{ }^{2} \\
0.5<\end{array}$ & $\begin{array}{l}{ }_{R}, \overline{w^{2}}{ }_{R} \\
<0.6\end{array}$ \\
\hline$\overline{v w}$ & $\begin{array}{r}\overline{v^{2}} \\
0.9<\end{array}$ & $\begin{array}{l}\overline{u v}_{S} \\
<1.0\end{array}$ & $\begin{array}{l}{\overline{v w_{R}}}, \bar{S}_{S}^{2}, \bar{v}^{2}{ }_{S} \\
0.2<\text { Co }<0.4\end{array}$ & $\begin{array}{c}{\overline{V_{R}}}^{2}, \bar{W}_{R}^{2} \\
\bar{u}_{R}^{2}, \bar{w}_{R}^{2} \\
0.5<\mathrm{Co}<0.6\end{array}$ & $\begin{array}{c}{\overline{S_{R}}}^{2},{\overline{S_{t}}}^{2} \\
{\overline{v^{2}}}_{R}, T K E_{R} \\
0.6<\text { Co }<0.7\end{array}$ & $\begin{array}{c}u_{* R}{ }^{2} \\
0.7<\text { Co }<0.8\end{array}$ \\
\hline$\overline{u t}$ & $\begin{array}{c}\overline{v t}_{R} \\
\text { Co }=-0.21\end{array}$ & $\begin{array}{c}\overline{w t}_{S} \\
\text { Co }=-0.99\end{array}$ & & $\begin{array}{r}\overline{w t} \\
\mathrm{Co}=\end{array}$ & & \\
\hline$\overline{v t}$ & & & & $\begin{array}{r}\overline{w t}_{R}, \overline{u t} \\
0.2<\mathrm{Cc}\end{array}$ & & \\
\hline$\overline{w t}$ & $\begin{array}{c}\overline{v t}_{R} \\
\text { Co }=0.21\end{array}$ & $\begin{array}{c}\overline{u t}_{S} \\
\text { Co }=-0.99\end{array}$ & & $\begin{array}{r}\overline{v t}_{R}, \\
0.2<\mathrm{Cc}\end{array}$ & $\begin{array}{l}\bar{t}_{R} \\
<0.4\end{array}$ & \\
\hline
\end{tabular}


to mean variables or those that can be measured conveniently. Properly scaled mean quantities form the input layers and turbulent fluxes are predicted at the output layer. Multilayer feedforward backpropagation network was used to find relationships between inputs and outputs while minimizing errors. The backpropagation algorithm is an iterative procedure that tries to modify weights based upon errors determined for all layers. Problems such as overfitting may arise in training a network, resulting in a poor generalization [10]. To avoid this, a simple optimization procedure was used to find a suitable network architecture containing an optimum number of hidden layers and neurons. It was speculated that in cases where the underlying relationships between variables are relatively simple, linear regression can outperform neural networks for relating turbulent and mean quantities. Therefore, it was necessary to understand the strength of linear or nonlinear correlations between different quantities before seeking complex relationships. Before creating a neural network, a systematic dimensional analysis was performed. All possible dimensionless groups containing two or three variables were built. Then, this analysis was used to distinguish between existing linear and nonlinear relationships. Moreover, high order polynomial functions were fit to the measured data to understand the severity of nonlinear relationships. This procedure provided us with a selection of quantities with known strong linear or nonlinear relationships.

\section{RESUlTS AND DisCUSSION}

\section{A. Two-variable Diemsionless Groups}

A systematic dimensional analysis identified all of the relevant two-variable dimensionless groups in table I. Those groups with linear correlation coefficients higher than 0.2 between turbulent fluxes and other quantities are shown. Some strong linear correlations were found at street level that were mostly related to other turbulent quantities such as variances along the street axis, $\overline{v^{2}}$. Significant linear correlation coefficients between different turbulent fluxes at street level, e.g. $\overline{u v}_{S}$ and $\overline{v w}_{S}$, provide an opportunity to find turbulent fluxes of interest by knowing the value of other fluxes, which may be accessible to determine. Among all momentum and heat fluxes, those with components in the vertical direction, i.e. $\overline{u w}$ and $\overline{w t}$, play important roles in the correct representation of the urban boundary layer. It is evident that there is a significant correlation between the momentum flux in the vertical direction, $\overline{u w}_{S}$, and other mean quantities including $\bar{U}_{S}{ }^{2}$ and $\bar{W}_{S}{ }^{2}$. Unlike at street level, the roof level turbulent fluxes did not exhibit high linear correlations with other variables. It is worth noting that there are still some linear relationships between vertical momentum fluxes, $\overline{v w}_{R}$ and $\overline{u w}_{R}$, and mean quantities with correlation coefficients of 0.4 to 0.6 . In comparison with flow within the canyon, wind speed and wind direction at roof-level are more likely to follow the flow patterns in the rural area. Linear correlations between rural wind speed, $\bar{S}_{t}{ }^{2}$, and momentum fluxes at roof level are shown in table I. Some other important correlations were also found, including relationships between $\overline{u v}_{R}, \overline{u w}_{R}$ and $\overline{v w}_{R}$, and squared mean velocity components within the canyon, $\bar{V}_{S}{ }^{2}$ and $\bar{U}_{S}{ }^{2}$. It is revealed that turbulent quantities at street level can be determined indirectly through mean quantities at the roof level and the rural site. Among all two-variable dimensionless groups, heat fluxes at roof-level only correlated with other heat fluxes at roof and street level. Higher order polynomial fits were also used to attempt to correlate the measured data for each two-variable dimensionless group. An example of the corresponding correlation coefficients is shown in Fig. 3. Comparing the value of correlation coefficients of linear and higher order polynomial functions, particularly groups containing $\bar{U}_{R}{ }^{2}, \bar{U}_{S}{ }^{2}$ and $\bar{W}_{S}{ }^{2}$, shows the existence of strong nonlinear relationships. For example, using a second order polynomial function to relate $\overline{u w}_{R}$ to $\bar{U}_{R}{ }^{2}$ increases the correlation coefficient by 178 percent. Fig. 4 shows how using the second order polynomial can improve the prediction of the relationship between $\overline{u w}_{R}$ and $\bar{U}_{R}{ }^{2}$. However, there was no significant change in correlation coefficients for groups containing $\bar{V}_{S}^{2}$ or $\bar{V}_{R}^{2}$. Likewise, using nonlinear functions for other vertical turbulent fluxes did not produce noticeable changes in correlation coefficient (not shown here). It can be concluded that nonlinear regression can significantly improve relationships between turbulent and some mean quantities. This highlights the importance of developing a proper nonlinear function which can describe nonlinear behavior of turbulent fluxes.

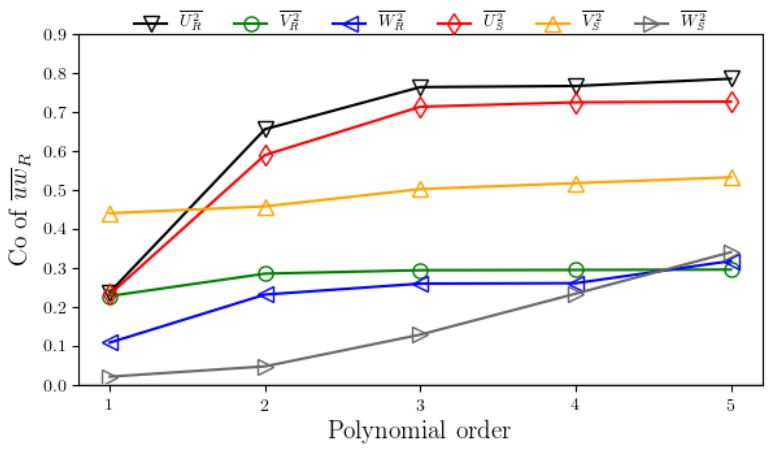

Figure 3: Correlation coefficient (Co) for different order polynomial regressions of the momentum flux

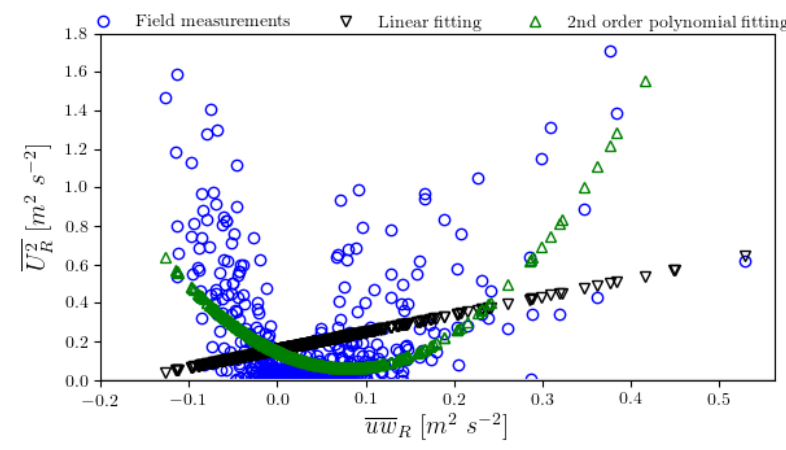

Figure 4: Comparison of linear and high order polynomial regression of momentum of flux

\section{B. Three-variable Diemsionless Group}

The same algorithm used above was also applied to threevariable dimensionless groups. Fig. 5 shows how higher order polynomial regressions can modify correlation coefficients. Vertical heat flux at roof level, $\overrightarrow{w t}_{R}$, had a strong nonlinear relationship with temperature differences between surface and adjacent atmosphere multiplied by vertical velocity (solid line). There are poor linear and nonlinear relationships between other heat flux (horizontal heat fluxes are not shown) and mean 
quantities. In this figure, $\bar{T}_{s r R}$ and $\bar{T}_{s r s}$ represents surface temperature at roof and street levels.

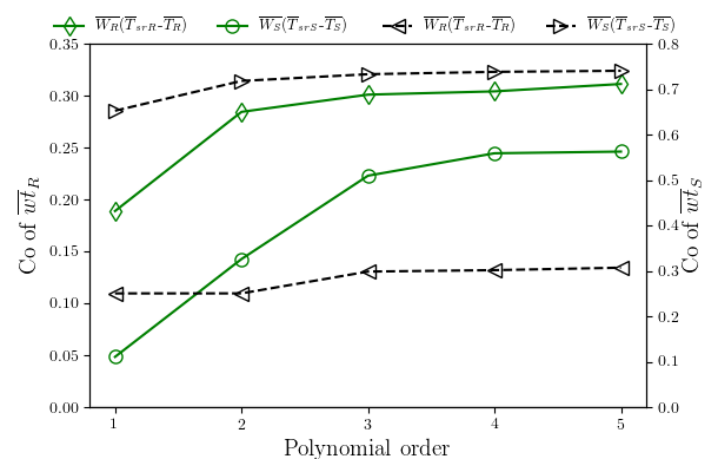

Figure 5: Correlation coefficient (Co) for different polynomial regressions of vertical heat flux; primary axis (solid lines) and secondary axis (dash lines)

\section{Developing a Multivariable Function}

The scaling studies provided insight into what mean quantities can be related to turbulent quantities. The unsteady and, to some extent, unpredictable nature of turbulent flows, particularly within a canyon, make turbulence modeling difficult and it seems unreliable to try to predict most features of turbulence using only one quantity. It has been suggested that multivariable functions can limit duplicity of solutions for a turbulence problem [11]. Thus, multivariable functions were developed using ANNs. Among all two and three-variable groups, those which exhibited high linear correlation coefficients between turbulent and mean quantities were chosen as candidates in the search for multivariable functions. The capability of an ANN to restrict and predict turbulent fluxes was evaluated by performing statistical

TABLE II. COMPARISON OF MULTIVARIABLE FUNCTIONS WITH SINGLE-VARIBLE FUNCTION OF TURBULENT FLUXES WITHIN CANYON

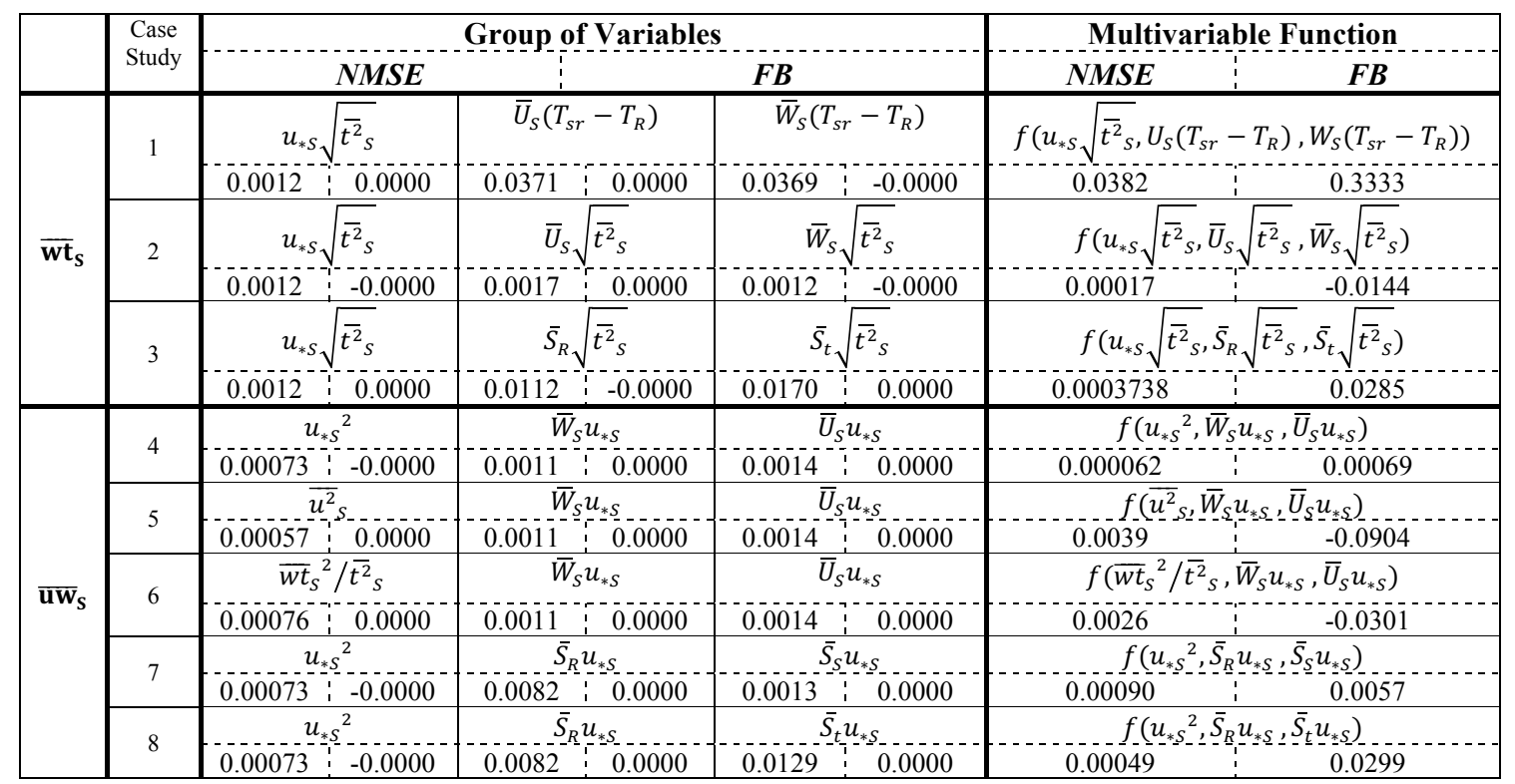

TABLE III. COMPARISON OF MULTIVARIABLE FUNCTIONS WITH SINGLE-VARIBLE FUNCTION OF TURBULENT FLUXE AT ROOF-LEVEL

\begin{tabular}{|c|c|c|c|c|c|c|}
\hline & \multirow{2}{*}{$\begin{array}{l}\text { Case } \\
\text { Study }\end{array}$} & \multicolumn{3}{|c|}{ Group of Variables } & \multicolumn{2}{|c|}{ Multivariable Function } \\
\hline & & NMSE & & $F B$ & $N M S E$ & $F B$ \\
\hline \multirow{4}{*}{$\overline{\mathbf{u w}}_{\mathrm{R}}$} & \multirow[b]{2}{*}{9} & $u_{* R}^{2}$ & $\bar{U}_{R} u_{* R}$ & $\bar{U}_{S} u_{* R}$ & \multicolumn{2}{|c|}{$f\left(u_{* R}^{2}, \bar{U}_{R} u_{* R}, \bar{U}_{S} u_{* R}\right)$} \\
\hline & & $\begin{array}{l:l}0.0081 & -0.0000\end{array}$ & $0.0022 \quad 0.0000$ & $0.0054, \quad 0.0000$ & \multicolumn{2}{|c|}{\begin{tabular}{c|c} 
& $\left(u_{* R}, v_{R} u_{* R}, v_{S} u_{* R}\right)$ \\
\hdashline 0.00036 & -0.0121
\end{tabular}} \\
\hline & \multirow{2}{*}{10} & $\overline{w t}_{R}^{2} / \bar{t}^{2}{ }_{R}$ & $\overline{u t}_{R}^{2} / \bar{t}^{2}{ }_{R}$ & $\bar{U}_{R} u_{* R}$ & \multicolumn{2}{|c|}{$f\left(\overline{w t}_{R}^{2} / \bar{t}_{R}^{2}, \overline{u t}_{R}^{2} / \bar{t}_{R}^{2}, \bar{U}_{R} u_{* R}\right.$} \\
\hline & & $\begin{array}{l:l}0.0082 & -0.0000\end{array}$ & $\begin{array}{l:l}0.0082 & 0.0000\end{array}$ & 0.0022 & 0.0011 & 0.0400 \\
\hline
\end{tabular}

analysis. Two metrics fractional bias (FB) and normalized mean square error (NMSE) were employed [12]. NMSE represents the overall scatter between observed and predicted quantities while FB quantifies the overall under- or over-estimation of measured quantities. Tables II and III show how efficiently and precisely ANNs can predict vertical turbulent fluxes. FB and NMSE were calculated for single-variable functions obtained from linear regressions of each induvial group of variables, and multivariable functions generated by ANN containing each group as an independent variable. Friction velocity, $u_{* S}=$ $\left(\overline{u w}_{S}^{2}+\overline{v w}_{S}^{2}\right)^{1 / 4}$, played an important role in scaling mean quantities leading to proper prediction of the turbulent fluxes. In comparison with linear regressions for the same case, an ANN often reduced NMSE by one order of magnitude. An ANN with input variables of $u_{* S} \sqrt{\overline{t^{2}}}{ }_{S}, \bar{S}_{R} \sqrt{\overline{t^{2}}}, \bar{S}_{t} \sqrt{\overline{t^{2}}}{ }_{S}$ showed 68 to 98 percent improvement in prediction of vertical heat flux at street level, $\overline{w t}_{s}$ compared to linear correlations. Employing ANNs also provided an opportunity to directly relate turbulent fluxes to horizontal rural velocity, $S_{t}$, with an acceptable accuracy. 
Notable improvements also occurred in ANN predictions of ${\overline{u w_{S}}}_{S}$ and $\overline{u w}_{R}$ with $f\left(u_{* S}{ }^{2}, \bar{W}_{S} u_{* S}, \bar{U}_{S} u_{* S}\right)$ and $f\left(u_{* R}{ }^{2}, \bar{U}_{R} u_{* R}, \bar{U}_{S} u_{* R}\right)$ as multivariable functions, respectively. On the other hand, FB deteriorated when an ANN was used to predict fluxes, but the results were still considered acceptable, meeting the range of acceptance criteria [13]. Figs. 6 and 7 compare predictions of hourly time series of turbulent fluxes using ANN and field measurements. These figures are based on case studies 3 and 9 , as referred to in Tables II and III, which represented significant improvement in prediction of relevant turbulent fluxes. Field data were collected at all wind speeds and directions, so no clear trend was expected for hourly flux time series. The level of agreement between observations and the ANN predictions is remarkable. This suggests that a well-trained ANN, such as built herein, can ingest, the inherently chaotic flow behavior within and above the canopy. However, ANNs failed to predict other turbulent fluxes, such as $\overline{u v}_{S}$ and $\overline{w t}_{R}$, using mean quantities. Additionally, as depicted in Tables II and III, linear regressions in cases 1 and 5 outperformed nonlinear multivariable functions.

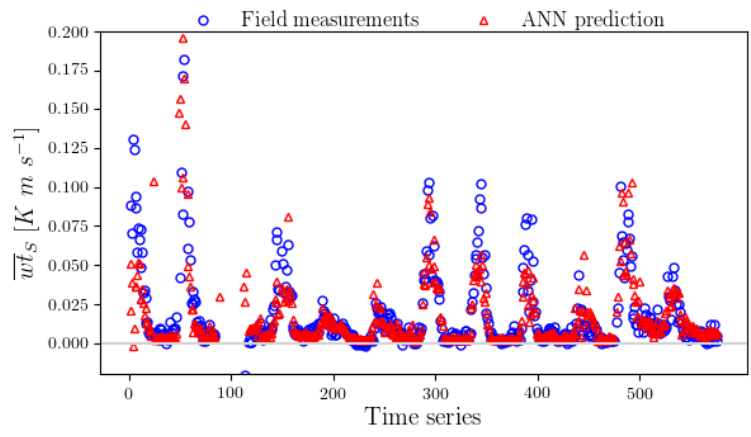

Figure 6: Comaprison of ANN results with measurement data of vertical heat flux within the canyon

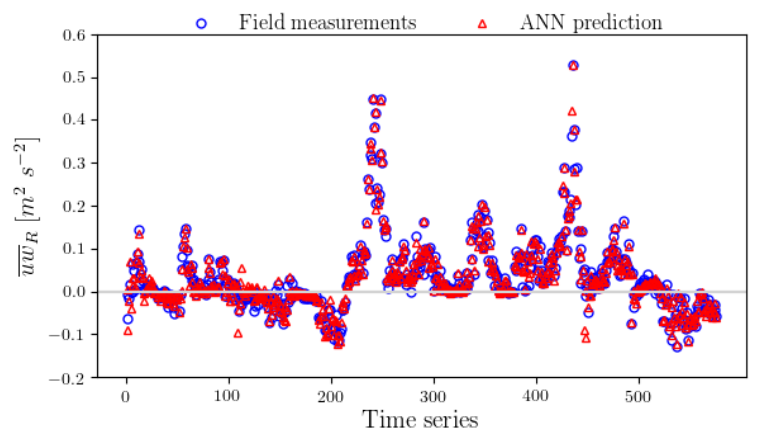

Figure 7: Comaprison of ANN results with measurement data of vertical momentum flux at roof-level

\section{CONCLUSIONS}

Systematic scaling analysis was performed to find relationships between turbulent and mean quantities within and above the urban canopy. Field data was collected under all wind directions, wind speeds, and thermal stability conditions from a street canyon within an urban canopy, and also a rural area, in Guelph, Ontario, Canada. Several approaches were used to establish relationships between turbulent and mean quantities. The significance of nonlinear behavior and relationships between quantities was evaluated by creating dimensionless groups of variables containing two or three quantities. High linear correlations for vertical momentum flux at both roof and street levels were found with mean wind speeds. This linear behavior is much more profound at street-level. Applying higher order polynomial functions to the field data revealed some strong nonlinear relationships. It was observed that using nonlinear functions can improve correlation coefficients significantly, for example, enhancing correlation coefficient by 178 percent for the relationship between $\overline{u w}_{R}$ and $\bar{U}_{R}{ }^{2}$. Similar results were found for the vertical heat flux. Additionally, it was found that artificial neural networks (ANNs) can be generated to predict such strong nonlinear relationships. The predictive capability of ANNs was evaluated for the current dataset by comparing NMSE and FB calculated by ANN and linear regressions. It was found that a well-trained ANN accurately predicted vertical momentum and heat fluxes within and above the canyon. However, in some cases linear regression outperformed ANN predictions. The different approaches are complementary to one another, and can be used in combination to further develop robust optimization algorithms for predicting turbulence quantities from mean meteorological values.

\section{ACKNOWLEDGEMENTS}

The lead author acknowledges the financial support of the NSERC Discovery Grant program. All authors thank the following individuals who helped with the urban microclimate field campaign: Denis Clement, Steve Nyman, Chris Duiker, Manuela Racki, Jeffrey Dafoe, Joanne Ryks, Ryan Smith, Stephen Stajkowski, and Baharam Gharabaghi.

\section{REFERENCES}

[1] Alberto Martilli, Jose Luis Santiago, Francisco Salamanca, "On the representation of urban heterogeneities in mesoscale models," Environ. Fluid Mech., 2015, 15:305-328

[2] R. E. Britter and S. R. Hanna, "Flow and dispersion in urban areas," Annu. Rev. Fluid Mech., 2003, 35: 469-496.

[3] S. E. Belcher, "Mixing and transport in urban areas," Philos. T. R. Soc. A., 2005,363(1837), 2947-2968.

[4] D. Zajic, H. J. S. Fernando, R. Calhoun, M. Princevac, M. J. Brown and E. R. Pardyjak, "Flow and turbulence in an urban canyon," J. Appl. Meteorol. Climatol., 2011, 50(1):203-223.

[5] R. B. Stull, "An introduction to boundary layer meteorology," 13. Springer Science \& Business Media, 1988.

[6] A. Venkatram and M. Princevac, "Using measurements in urban areas to estimate turbulent velocities for modeling dispersion," Atmos. Environ., 2008.

[7] M. Roth and T. Oke, "Turbulent transfert: relationships over an urban surface. I: spectral characteristics,” Quart. J. Roy. Meteorol. Soc., 1993, $119,1071-1104$.

[8] L. Quan and F. Hu, "Relationship between turbulent flux and variance in the urban canopy," Meteorol. Atmos. Phys., 2009, 104:29-36.

[9] A. Aliabadi, M. Moradi, D. Clement, W. D. Lubitz and B. Gharabaghi, "Flow and temperature dynamics in an urban canyon under all wind directions, wind speeds, and thermal stability conditions," Environ. Fluid Mech., (submitted).

[10] G. Dreyfus, "Neural networks: methodology and applications," Springer, Berlin, Heidelberg, 2005

[11] S. J. Linz and J.C. Sprott, "Elementary chaotic flow," Phys. Lett. A, 1999, 259:240-245.

[12] A. A. Aliabadi, E. S. Krayenhoff, N. Nazarian, L. W. Chew, P. R. Armstrong, A. Afshari and L. K. Norford, "Effects of roof-rdge roughness on air temperature and pollutant concentration in urban canyons," Bound.Layer Meteorol., 2017, 164:249-279.

[13] J. C. Chang and S. R. Hanna, "Technical descriptions and users guide for the boot statistical model evaluation software package," version 2.0, 200 